\title{
Cleavage of the ether bond by electron impact: differences between linear ethers and tetrahydrofuran $\dagger$
}

\author{
Bogdan C. Ibănescu, Olivier May and Michael Allan
}

\begin{abstract}
Dissociative electron attachment (DEA) to diethyl ether yielded primarily the $\mathrm{C}_{2} \mathrm{H}_{5} \mathrm{O}^{-}$ion, with a strong Feshbach resonance band at $9.1 \mathrm{eV}$ and a weaker shape resonance band at $3.89 \mathrm{eV}$. Very similar spectra were obtained for dibutyl ether, with $\mathrm{C}_{4} \mathrm{H}_{9} \mathrm{O}^{-}$bands at 8.0 and $3.6 \mathrm{eV}$. Some of these primary ions subsequently lost $\mathrm{H}_{2}$ and yielded weaker signals of the $\mathrm{C}_{2} \mathrm{H}_{3} \mathrm{O}^{-}$and $\mathrm{C}_{4} \mathrm{H}_{7} \mathrm{O}^{-}$ ions. In contrast, DEA to the cyclic ether tetrahydrofuran (THF) yielded mainly a fragment of mass 41 , presumably deprotonated ketene, at $7.65 \mathrm{eV}$. The low-energy band was missing in THF. $\mathrm{H}^{-}$with two bands at 6.88 and $8.61 \mathrm{eV}$, and an ion of mass 43 (presumably deprotonated acetaldehyde) with two bands at 6.7 and $8.50 \mathrm{eV}$ were also observed. We propose that in the primary DEA step the $\mathrm{C}-\mathrm{O}$ bond is cleaved in both the open-chain and the cyclic ethers. In the open-chain ethers the excess energy is partitioned between the (internal and kinetic) energies of two fragments, resulting in an $\mathrm{RO}^{-}$ion cool enough to be observed. The ${ }^{\bullet} \mathrm{CH}_{2}\left(\mathrm{CH}_{2}\right)_{3} \mathrm{O}^{-}$ion resulting from cleavage of the $\mathrm{C}-\mathrm{O}$ bond in THF contains the entire excess energy (more than 6 $\mathrm{eV}$ at an electron energy of $7.65 \mathrm{eV}$ ) and is too short-lived with respect to further dissociation and thermal autodetachment to be detected in a mass spectrometer. These findings imply that there could be a substantial difference between the fragmentation in the gas phase described here and fragmentation in the condensed phase where the initially formed fragments can be rapidly cooled by the environment.
\end{abstract}

\section{Introduction}

Electron interactions with the cyclic ether tetrahydrofuran (THF) have been studied because THF serves as a convenient model for the sugar ring in the DNA backbone in connection with radiation damage. ${ }^{1}$ Of particular importance in respect to this application are studies in the condensed phase. Lepage et l. $^{2}$ studied resonances (i.e., temporary anion states which act as intermediates in the electron-induced degradation) in THF by means of resonant vibrational excitation (VE) in thinfilm THF and detected at least three resonances, located near 4, 7.5 , and $10 \mathrm{eV}$. Antic et al. ${ }^{1}$ studied the electron-induced yield of negative ions from frozen THF and observed formation of $\mathrm{H}^{-}$at $10 \mathrm{eV}$, attributed to a core excited resonance. Electron energy loss spectra (EELS), in particular the observation of an $\left(n, \pi^{*}\right)$ transition, revealed neutral degradation products containing carbonyl groups. Antic et al. ${ }^{3}$ reported a resonance at $23 \mathrm{eV}$ which decayed into a highly excited state undergoing a further dipolar dissociation. The formation of aldehydes from THF frozen on a $\mathrm{Kr}$ substrate was studied in detail by Breton et al. ${ }^{4}$ and Jäggle et al. ${ }^{5}$ by means of vibrational and electronic EELS of the products. A strong rise of aldehyde production was observed from about $6 \mathrm{eV}$ upward and was correlated to $\left(n, \sigma_{\mathrm{CO}}^{*}\right)$ electronic excitation threshold of THF, together with core-excited resonances around

Department of Chemistry, University of Fribourg, Chemin du Musée, 9, CH-1700 Fribourg, Switzerland
9 and $10 \mathrm{eV}$. The absolute cross section reached a value of $\sim 6000$ $\mathrm{pm}^{2}$ above $11 \mathrm{eV}$. A small feature found around $3 \mathrm{eV}$ was proposed to result from a $\sigma^{*}$ shape resonance. The formation of olefins and $\mathrm{CO}$ were identified in the later study. Electron trapping processes in condensed THF were studied quantitatively by Park et al. ${ }^{6}$ The charge trapping cross section for $6-9 \mathrm{eV}$ electrons was found to have an upper limit of $40 \mathrm{pm}^{2}$, much smaller than the aldehyde production cross section mentioned above, leading to the conclusion that the major part of THF degradation has electronic excitation and not dissociative electron attachment (DEA) as the primary step. A much larger trapping cross section ( $\sim 4000 \mathrm{pm}^{2}$ for 2 monolayers), strongly dependent on the quantity of deposited THF, and proceeding via intermolecular stabilization, was found in the $0-0.1 \mathrm{eV}$ range.

The condensed phase work was complemented by gas phase DEA fragmentation studies performed by Sulzer et al. ${ }^{7}$ Of particular importance is the quantitative study of Aflatooni et al. ${ }^{8}$ who found two DEA bands, at 6.2 and $8 \mathrm{eV}$, with a surprisingly small cross section, $1.5 \mathrm{pm}^{2}$, about a factor 30 less than that in the 3-hydroxy substituted THF.

Indispensable for the global understanding of the electroninduced processes are also gas phase studies not directly involving chemical change. Zecca et al. ${ }^{9}$ and Możejko et al. ${ }^{10}$ measured the absolute total cross section for electron scattering by gas-phase THF (Zecca et al. reported also the positron cross section). The elastic and/or vibrational excitation differential cross sections were measured by Milosavljević et al., ${ }^{11}$ Colyer et al., ${ }^{12}$ Dampc et al. ${ }^{13,14}$ and in this laboratory. ${ }^{15}$ The studies identified broad resonant bands around 6.2 
and $10.8 \mathrm{eV}$, assigned to two or more overlapping shape resonances. Indication of an additional resonant process around $2.6 \mathrm{eV}$ was reported in the last study.

High-level scattering calculations of the elastic and/or momentum-transfer cross sections in THF were reported by Trevisan et al., ${ }^{16}$ Winstead and McKoy ${ }^{17}$ and Tonzani and Greene. ${ }^{18}$ Bouchiha et al. ${ }^{19}$ also calculated the electronically inelastic cross section and the energies of a number of coreexcited (Feshbach) resonances.

The present work reports DEA spectra of diethyl ether, dibutyl ether and THF with the aim of gaining insight into how susceptible is the ether bond to cleavage by electron impact, and understanding the differences between cyclic and open-chain ethers. The similarities of, and differences between, the cleavage of the bond $\mathrm{O}-\mathrm{C}$ in ethers, and the $\mathrm{O}-\mathrm{C}$ and $\mathrm{O}-\mathrm{H}$ bonds in alcohols are also discussed.

\section{Methods}

The dissociative electron attachment spectrometer used to measure the yield of mass-selected stable anions as a function of electron energy has been described previously. ${ }^{20-22}$ It employs a magnetically collimated trochoidal electron monochromator $^{23}$ to prepare a beam of quasi-monoenergetic electrons, which is directed into a target chamber filled with a quasi-static sample gas. Fragment anions are extracted at $90^{\circ}$ by a three-cylinder lens and directed into a quadrupole mass spectrometer. The energy scale was calibrated on the onset of the $\mathrm{O}^{-} / \mathrm{CO}_{2}$ signal at $4.0 \mathrm{eV}$. The electron current was around $200 \mathrm{nA}$ and the resolution about $150 \mathrm{meV}$. Photoelectron (PE) spectra were recorded with a modified Perkin Elmer PS18 HeI photoelectron spectrometer. Threshold energies for various fragmentations were calculated as the differences of the total energies of the products and the targets at $0 \mathrm{~K}$, corrected for the zero point vibrational energy, using the density functional theory (DFT) B3LYP/6-311+G(2df,2p) model, ${ }^{24}$ applied already in our previous study of alcohols. ${ }^{22}$

\section{Results and discussion}

Fig. 1-3 show the DEA spectra of THF and two linear ethers related to it. Diethyl ether has the same total number of carbon atoms as THF. Dibutyl ether is in a certain sense even closer related to THF because the alkyl group is four carbon atoms long for both. Qualitative indications of the relative signal intensities are given by the count rates, which were normalized to a current of $200 \mathrm{nA}$ and a main chamber pressure of $2 \times 10^{-6} \mathrm{mbar}$. The pressure is an uncorrected Penning gauge reading, but the gauge sensitivity may be expected to be comparable for the compound studied here, particularly for diethyl ether and THF, which have the same number of heavy atoms. The relative intensities may thus be compared qualitatively even between the compounds. The vacuum chamber is equipped with a liquid nitrogen trap, which was generally filled during the measurements, resulting in a main chamber pressure between $1 \times 10^{-7}$ and $5 \times 10^{-7}$ mbar-barely measurable with the Penning gauge. The count rate measurements were consequently taken without liquid nitrogen in the trap to yield higher, more accurate pressure readings. The $\mathrm{H}^{-}$

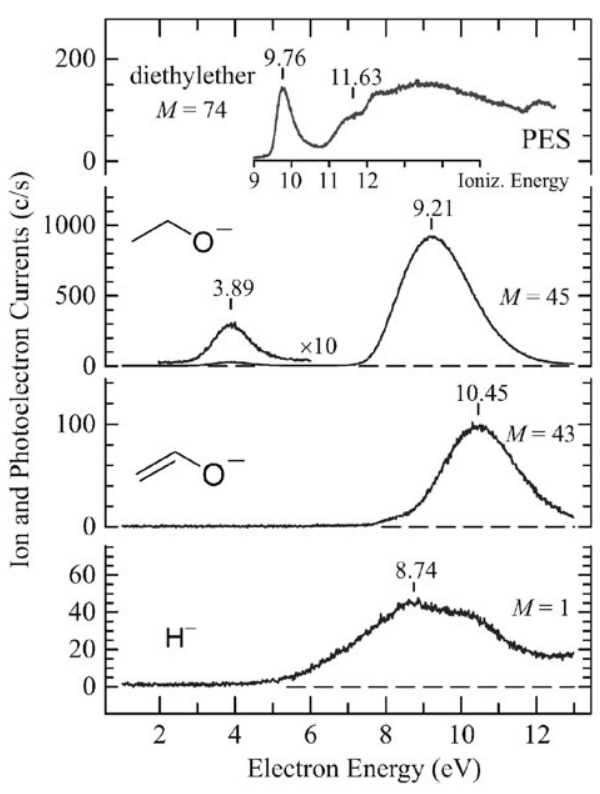

Fig. 1 The HeI photoelectron spectrum (top, shown shifted by $-4.5 \mathrm{eV}$ ), and the yields of the fragments with the masses indicated, for diethyl ether.

intensity can not be compared with the intensity of the other fragments, however, because the transmissivity of the ion lens and the quadrupole mass filter may be expected to be substantially different for this very light ion, which is harder to guide in the magnetic field of the trochoidal monochromator.

The strongest signal from diethyl ether has the mass of 45, and may be assigned with confidence to the ethanolate anion $\mathrm{CH}_{3} \mathrm{CH}_{2} \mathrm{O}^{-}$, resulting from the cleavage of one $\mathrm{C}-\mathrm{O}$ bond. The process is thus related to the $\mathrm{C}-\mathrm{O}$ bond cleavage in many alcohols (leading to $\mathrm{OH}^{-}$formation), with a band at a similar energy. 22,25,26

An indication of the expected energies of Feshbach resonances can be obtained from ionization energies, measured by photoelectron (PE) spectroscopy. The prediction is based on the observation that the energy difference between the ${ }^{2}\left(\psi_{i}^{-1}\right)$ grandparent cation state (where $\psi_{i}$ is the $i$-th occupied molecular orbital) and the ${ }^{2}\left(\psi_{i}, 3 \mathrm{~s}^{2}\right)$ Feshbach resonance is about the same $(4.5 \mathrm{eV})$ for a wide range of molecules and even for rare gases (references 22, 27, 28 and references therein). This observation is rationalized by the large spatial extent of the doubly occupied 3s Rydberg-like orbital which consequently does not strongly penetrate the positive ion core. The bands in

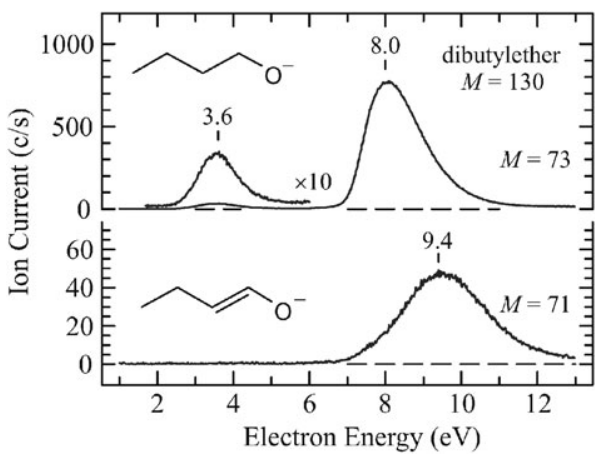

Fig. 2 DEA spectra of dibutyl ether. 


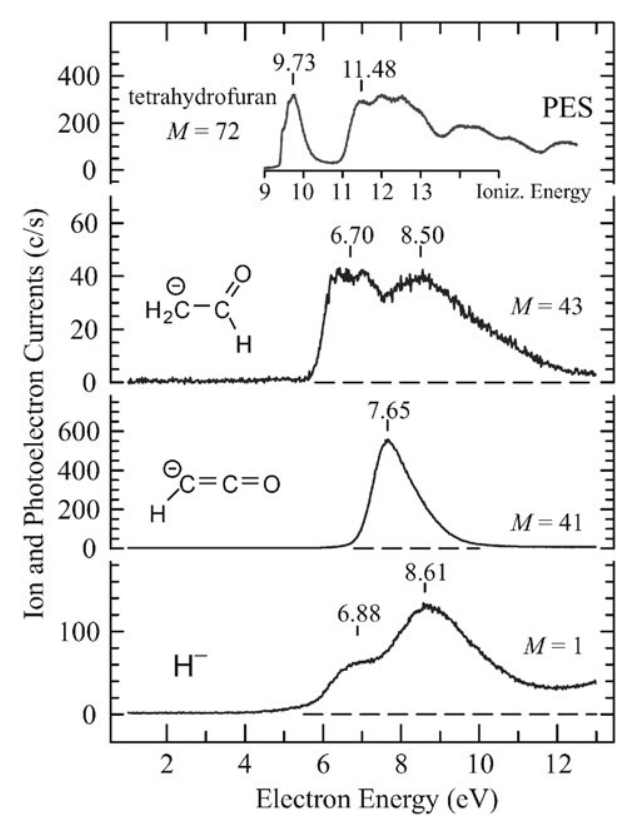

Fig. 3 The HeI photoelectron spectrum (top, shown shifted by $-4.5 \mathrm{eV}$ ) and DEA spectra of tetrahydrofuran.

the shifted PE spectrum in Fig. 1 and 3 thus indicate the expected positions, and also Franck-Condon widths, of the Feshbach resonances of the type ${ }^{2}\left(\psi_{i}, 3 \mathrm{~s}^{2}\right)$, with a hole in the normally occupied $\psi_{i}$ valence orbital and a double occupation of a Rydberg-like 3s orbital.

The $9.21 \mathrm{eV}$ band in Fig. 1 is at a much higher energy, and is much wider, than would be expected for the low-lying Feshbach resonances ${ }^{2}\left(\psi_{\mathrm{O}}, 3 \mathrm{~s}^{2}\right)$ or ${ }^{2}\left(\overline{\mathrm{n}}_{\mathrm{O}}, 3 \mathrm{~s}^{2}\right)$, associated with the 9.76 and $11.63 \mathrm{eV}$ bands in the PE spectrum shown in the same figure. At the position in the PE spectrum corresponding to the 9.21 eV DEA band there is the ' $\sigma$-mountain', consisting of many broad overlapping bands corresponding to ionizations from $\sigma$ orbitals localized on the $\mathrm{C}-\mathrm{C}$ and $\mathrm{C}-\mathrm{H}$ bonds, and we assign the $9.21 \mathrm{eV}$ band to one or several of the corresponding Feshbach resonances. High-lying shape resonances are also expected in this energy range, but these resonances generally have an autodetachment rate too fast to permit dissociation and we consequently favor the assignment to Feshbach resonances, although a varying degree of mixing of shape and Feshbach configurations, analogous to mixing of valence and Rydberg states in neutral molecules ${ }^{29,30}$ is probable.

There is little doubt that ${ }^{2}\left(\mathrm{n}_{\mathrm{O}}, 3 \mathrm{~s}^{2}\right)$ or ${ }^{2}\left(\overline{\mathrm{n}}_{\mathrm{O}}, 3 \mathrm{~s}^{2}\right)$ Feshbach resonances, with holes in the oxygen lone pair orbitals, occur around 5.2 and $7 \mathrm{eV}$ in diethyl ether-but they do not lead to observable dissociation, in contrast to the H-loss from the alcohols. This indicates that they are, in contrast to the corresponding resonances in the alcohols, not suitably predissociated by a repulsive valence resonance with an antibonding $\sigma^{*}$ orbital occupation (shape or valence core excited). It is interesting to note that a related observation has been made for the parent Rydberg states. Robin ${ }^{29,30}$ analyzed term energies and band shapes in VUV spectra and recognized that in water and the alcohols the lowest ${ }^{1}\left(n_{\mathrm{O}}, 3 \mathrm{~s}\right)$ parent Rydberg states are unusually low in energy and the bands are broad and structureless - whereas the corresponding photoelectron bands are narrow with sharp vibrational structure. In contrast, the lowest Rydberg bands in the ethers behaved normally. Robin concluded that the ${ }^{1}\left(n_{\mathrm{O}}, 3 \mathrm{~s}\right)$ Rydberg states are strongly perturbed by the conjugate ${ }^{1}\left(\mathrm{n}_{\mathrm{O}}, \sigma^{*}\right)$ valence promotions in the alcohols and water, but that this perturbation is not significant in the ethers.

The fragment with mass 43 is probably formed by loss of $\mathrm{H}_{2}$ from the initially formed (hot) ethanolate anion. The $\mathrm{H}^{-}$band is very broad and hard to assign to individual Feshbach resonances.

The weak band at $3.89 \mathrm{eV}$ (mass 45) is too low for a Feshbach resonance and must be due to a $\sigma^{*}$ shape resonance. We calculated the threshold for this process to be $1.7 \mathrm{eV}$, using the model validated on alcohols. ${ }^{22}$ The facts that the $3.89 \mathrm{eV}$ band peaks far above the threshold and that it does not have a vertical onset indicate that, in contrast to the similar lowenergy bands found for alcohols, ${ }^{22}$ there is an activation barrier on the dissociation path.

The DEA spectra of dibutyl ether in Fig. 2 are similar to those of diethyl ether and indicate an efficient cleavage of the $\mathrm{C}-\mathrm{O}$ bond with both the low energy $(3.6 \mathrm{eV})$ and the high energy $(8 \mathrm{eV})$ bands. We calculate the threshold for the $M=$ 73 ion formation to be $1.6 \mathrm{eV}$, indicating an energy barrier on the dissociation path leading to the $3.6 \mathrm{eV}$ band, similarly to the diethyl ether case. The $9.4 \mathrm{eV} M=71$ band is probably due to a subsequent loss of an $\mathrm{H}_{2}$ molecule from the primary $M=73$ anion. There is one important difference, however, the energy of the upper $\mathrm{RO}^{-}$band dropped by $1.2 \mathrm{eV}$ (from 9.2 to $8 \mathrm{eV}$ ) with respect to diethyl ether, indicating a fairly strong dependence on the size of the alkyl substituent. The $1.2 \mathrm{eV}$ energy difference is similar to the difference of the ionization energies of ethane and $n$-butane (about $1 \mathrm{eV}$ ), ${ }^{31}$ in line with the assignment of the ether DEA bands to Feshbach resonances associated with ionizations from orbitals of the alkyl groups.

The most intense fragment from THF has the mass of 41 with a peak at $7.65 \mathrm{eV}$, in agreement with the previous work of Sulzer et $a .^{7}$ A fragment with this mass is frequently found in DEA (see, for example, reference 32) and probably has the stable structure of deprotonated ketene. We did not find the ions with masses 70 and 72 reported at $1.25 \mathrm{eV},{ }^{7}$ however. The $m / e=72$ ion corresponds to the full molecular mass, and detection of ions with the full molecular mass is very rare in DEA at non-thermal energies, because such ions tend to thermally lose the electron or to dissociate as will be detailed further below.

Comparison with the absolute (but not mass resolved) spectrum of Aflatooni et al. ${ }^{8}$ is less straightforward. The two DEA bands at 6.2 and $8 \mathrm{eV}$ which they report resemble the two bands which we observe for the fragments with masses 1 and 43 . The present $7.65 \mathrm{eV}$, mass 41 band could contribute to the second band in the spectrum of Aflatooni et al., ${ }^{8}$ which could be reduced in intensity in their spectrum by the onset of the positive ion current. Finally, our instrument could underestimate the $\mathrm{H}^{-}$intensity. With these points in mind, the present spectra are compatible with the results of Aflatooni et al.

The two bands in the yield of $\mathrm{H}^{-}$in Fig. 3 could have the same origin as the $10 \mathrm{eV}$ band and $7.3 \mathrm{eV}$ shoulder in the $\mathrm{H}^{-}$ yield from frozen THF reported by Antic et al. ${ }^{1}$ It is plausible to assume that the present two bands are shifted to higher energies and broadened by the action of the condensed media on the spatially large Rydberg-like orbitals of the Feshbach resonances. Finally a fragment with mass 43 , whose structure could 


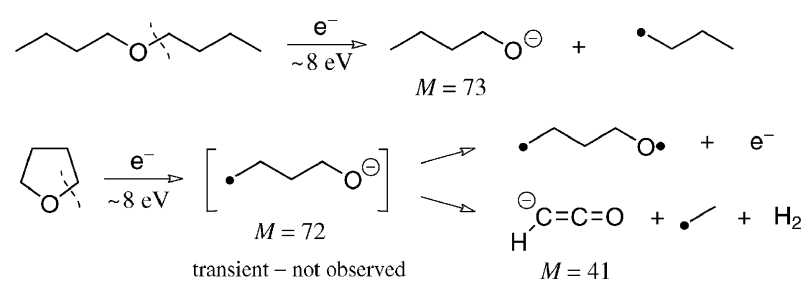

Fig. 4 Hypothetical electron-induced reactions. The butanolate anion formed in the upper reaction may be cooled by depositing part of the excess energy into vibrations of the neutral butyl fragment and the kinetic energy of the products. The $M=72$ fragment in the lower scheme does not have this possibility and the high internal energy makes it too short-lived to be detected mass-spectrometrically - only the $M=41$ fragment is observed.

be deprotonated acetaldehyde, is observed with weak intensity. Its spectrum has two bands with energies similar to those in the $\mathrm{H}^{-}$spectrum. It could thus be that the same resonant mechanisms which lead to $\mathrm{H}^{-}$also lead to $\mathrm{H}$ and a hot $(M-1)^{-}$ion, which then dissociates further to the mass 43 fragment.

The present spectra of diethyl ether and dibutyl ether show that the $\mathrm{C}-\mathrm{O}$ bond is not inert to cleavage by electron impact but breaks to yield the $\mathrm{R}-\mathrm{O}^{-}$anion, both at low energy, via a shape resonance, and at high energy, via a Feshbach resonance. The process resembles the cleavage of the $\mathrm{O}-\mathrm{H}$ bond in the alcohols with the difference that activation energy is necessary at the low energy band and dissociation occurs only from the higher excited Feshbach resonances for the high energy bands.

There is no plausible reason why the $\mathrm{C}-\mathrm{O}$ bond in THF should not break in the same way as in the linear ethers - as proposed in the scheme in Fig. 4. In fact, the $7.65 \mathrm{eV}$ (mass 41) band in THF is very similar to the $8.0 \mathrm{eV}$ (mass 73) band of dibutyl ether both in terms of energy and of band shape, suggesting that the initial step of the resonant dissociation is essentially the same in both cases. The principal difference is that in the linear ethers the products may be cooled by transferring part of the excess energy into the kinetic energy of the fragments and the internal degrees of freedom (vibrational and possibly even electronic excitation) of the neutral fragment, whereas in THF the entire excess energy remains in the negative ion product. An excess energy of $6.4 \mathrm{eV}$ is obtained for $7.7 \mathrm{eV}$ incident electrons using our calculated threshold for the - $\mathrm{CH}_{2}\left(\mathrm{CH}_{2}\right)_{3} \mathrm{O}^{-}$ion formation of $1.3 \mathrm{eV}$. The ${ }^{\bullet} \mathrm{CH}_{2}\left(\mathrm{CH}_{2}\right)_{3} \mathrm{O}^{-}$ ion thus necessarily has enough energy to either dissociate further or to lose an electron by autodetachment. It is consequently too short-lived to be observed in a mass spectrometer which involves a flight time of the order of $10 \mu \mathrm{s}$.

The present lack of observation of the $(M)^{-}$ion from THF is in line with the fact that there are only few known cases where the attachment of a non-thermal electron leads to an anion with the full molecular mass $M$ sufficiently long-lived to be detected in a mass spectrometer. A notable example is the p-benzoquinone where the attachment of $1.4 \mathrm{eV}$ electrons leads to a long-lived anion with the full molecular mass. ${ }^{33,34}$ A more recently discovered case is $\mathrm{C}_{60}$ (reference 35 and references therein) - but it is not comparable to the present case because the long-term electron attachment occurs over a very wide range of energies, not via a specific resonance. A relevant example where a search for a long-lived anion with

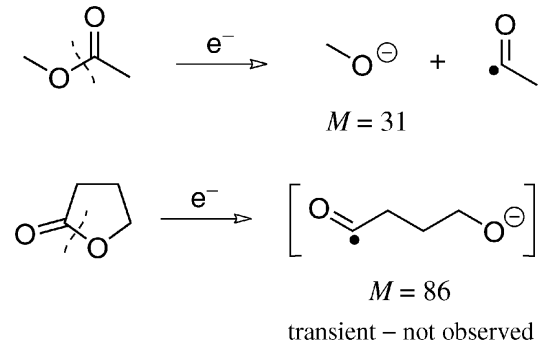

Fig. 5 Electron-induced reactions of methyl acetate and $\gamma$-butyrolactone. The methanolate anion was observed with a large intensity whereas the $M=86$ anion from $\gamma$-butyrolactone was not observed. ${ }^{20,32}$

the full molecular mass failed is $\gamma$-butyrolactone, ${ }^{20,32}$ illustrated in the scheme in Fig. 5. It is related to the present case in the sense that the ester bond was shown to break readily in the linear esters and that the cleavage of the ester bond in the cyclic $\gamma$-butyrolactone should lead to an openchain anion with the full molecular mass. Such an $(M)^{-}$ion was, however, not observed in the experiment, with the same explanation being invoked as in the present case for THF.

The $3.8 \mathrm{eV}$ band of diethyl and dibutyl ethers does not have an analogy in the THF spectrum in our measurements, although it is energetically possible. Even at this energy there is no plausible reason why the $\mathrm{C}-\mathrm{O}$ bond should behave very differently in the cyclic and the open-chain ethers. We presume that the $\mathrm{C}-\mathrm{O}$ bond does break in THF, but the resulting - $\mathrm{CH}_{2}\left(\mathrm{CH}_{2}\right)_{3} \mathrm{O}^{-}$ion is metastable in respect to autodetachment, which would in this case require that the ring is re-closed. The initial ${ }^{\bullet} \mathrm{CH}_{2}\left(\mathrm{CH}_{2}\right)_{3} \mathrm{O}^{-}$ion could be stabilized in the condensed phase, however, and give rise to the weak process reported around $3 \mathrm{eV}$ by Breton et al. ${ }^{4}$

\section{Conclusions}

Dissociative electron attachment to the linear diethyl and dibutyl ethers on the one hand and the cyclic ether THF on the other hand yield very different types of fragments in the Feshbach resonance energy region $(6-12 \mathrm{eV})$ - but we conclude that the primary step, a cleavage of the $\mathrm{C}-\mathrm{O}$ bond, is the same in both cases.

We propose that the different appearance of the detected fragmentation patterns stems from the fact that the primary fragment from, for example, dibutyl ether, the butanolate anion $\mathrm{CH}_{3}\left(\mathrm{CH}_{2}\right)_{3} \mathrm{O}^{-}$, can be 'cooled' by depositing part of the excess energy of the reaction (which is about $6.4 \mathrm{eV}$ for an attachment of an $8 \mathrm{eV}$ electron) into the kinetic energy of the fragments and the internal energy of the neutral fragment, the butyl radical. At least some of the butanolate anions are produced with sufficiently low internal energy and consequently long lifetime to be detected in the mass spectrometer. (Part of the anions fragment further by losing an $\mathrm{H}_{2}$ molecule, another part may thermally detach an electron and not be detected at all.)

In contrast, cleavage of the $\mathrm{C}-\mathrm{O}$ bond in THF does not lead to two fragments, but only one ion, ${ }^{\bullet} \mathrm{CH}_{2}\left(\mathrm{CH}_{2}\right)_{3} \mathrm{O}^{-}$, which necessarily retains all of the excess energy, making it too short-lived with respect to further dissociation, and probably also thermal autodetachment, to be detected in a mass spectrometer. The 
subsequent dissociation appears to lead primarily to a fragment with a mass of 41 , probably deprotonated ketene.

These findings imply that the fragmentation pattern of THF in the condensed phase could be quite different from the gas phase, because the initially formed ${ }^{\bullet} \mathrm{CH}_{2}\left(\mathrm{CH}_{2}\right)_{3} \mathrm{O}^{-}$fragment could be cooled sufficiently rapidly in the condensed phase to quench further dissociation and autodetachment. The small value of the electron trapping cross section in the 6-9 $\mathrm{eV}$ range measured by Park et al. ${ }^{6}$ indicates that this process does not have a very large cross section even in the condensed phase, however.

The $\mathrm{C}-\mathrm{O}$ bond is cleaved also by $\sim 3.5 \mathrm{eV}$ electrons in the linear ethers, via a $\sigma^{*}$ shape resonance, and an energy barrier is involved. It is well possible that this $\sim 3.5 \mathrm{eV}$ cleavage occurs also in THF but the resulting anion is metastable toward the reverse reaction of ring-closure and subsequent autodetachment.

The indications of metastability of the fragment anions are indirect in the present work, but it may be worth noting that there is a known case where metastability of $\mathrm{C}_{3}{ }^{-}$fragment anion, formed by DEA to acetaldehyde via a Feshbach resonance, has been measured directly by delayed coincidence of detached electrons. ${ }^{36}$ A direct observation of metastability of negative ion fragments was recently reported in DEA to amino acids. ${ }^{37}$

The metastability of the fragment anions could have the consequence that different instruments, with different time intervals between ion formation and detection of the anions, could yield different DEA cross section values, and possibly even different DEA spectra shapes.

The present work further reveals an important difference in the dissociation patterns of Feshbach resonances in alcohols and in ethers. Whereas the $\mathrm{O}-\mathrm{H}$ bond cleavage in alcohols proceeds already from the lowest Feshbach resonance (with hole in the oxygen lone pair orbital), the $\mathrm{C}-\mathrm{O}$ bond cleavage in ethers proceeds only from higher-lying Feshbach resonances with holes in the $\sigma_{\mathrm{C}-\mathrm{C}}$ and $\sigma_{\mathrm{C}-\mathrm{H}}$ orbitals. From this observation we conclude that the lowest Feshbach resonance in the alcohols is predissociated by a repulsive resonant state with a $\sigma^{*}$ orbital occupation, but no such suitable repulsive surface is available in the ethers. An interesting related conclusion was made for the parent states of the Feshbach resonances, the Rydberg states of the neutral molecules, where strong Rydberg- $\sigma^{*}$ valence mixing was deduced for alcohols (and water) but not for ethers. ${ }^{29,30}$

A difference in the dissociation pattern of ethers and alcohols is also observed in the low energy region $(1-5 \mathrm{eV})$, where alcohols dissociate without an activation barrier, whereas substantial barrier is found in the ethers.

\section{Acknowledgements}

This research is part of project No. 200020-113599/1 of the Swiss National Science Foundation.

\section{References}

1 D. Antic, L. Parenteau, M. Lepage and L. Sanche, J. Phys. Chem. $B, 1999,103,6611$.

2 M. Lepage, S. Letarte, M. Michaud, F. Motte-Tollet, M.-J. HubinFranskin, D. Roy and L. Sanche, J. Chem. Phys., 1998, 109, 5980.

3 D. Antic, L. Parenteau and L. Sanche, J. Phys. Chem. B, 2000, 104, 4711.

4 S.-P. Breton, M. Michaud, C. Jäggle, P. Swiderek and L. Sanche, J. Chem. Phys., 2004, 121, 11240.
5 C. Jäggle, P. Swiderek, S.-P. Breton, M. Michaud and L. Sanche, J. Phys. Chem. B, 2006, 110, 12512.

6 Y. S. Park, H. Cho, L. Parenteau, A. D. Bass and L. Sanche, J. Chem. Phys., 2006, 125, 074714.

7 P. Sulzer, S. Ptasiñska, F. Zappa, B. Mielewska, A. R. Milosavljević, P. Scheier, T. D. Märk, I. Bald, S. Gohlke, M. A. Huels and E. Illenberger, J. Chem. Phys., 2006, 125, 044304.

8 A. Aflatooni, A. M. Scheer and P. D. Burrow, J. Chem. Phys., 2006, 125, 054301.

9 A. Zecca, C. Perazzolli and M. Brunger, J. Phys. B: At., Mol. Opt. Phys., 2005, 38, 2079.

10 P. Możejko, E. Ptasińska-Denga, A. Domaracka and C. Szmytkowski, Phys. Rev. A, 2006, 74, 012708.

11 A. R. Milosavljević, A. Giuliani, D. Sević, M.-J. Hubin-Franskin and B. P. Marinković, Eur. Phys. J. D, 2005, 35, 411.

12 C. J. Colyer, V. Vizcaino, J. P. Sullivan, M. J. Brunger and S. J. Buckman, New J. Phys., 2007, 9, 41.

13 M. Dampc, A. R. Milosavljević, I. Linert, B. P. Marinković and M. Zubek, Phys. Rev. A, 2007, 75, 042710.

14 M. Dampc, I. Linert, A. R. Milosavljević and M. Zubek, Chem. Phys. Lett., 2007, 443, 17.

15 M. Allan, J. Phys. B: At., Mol. Opt. Phys., 2007, 40, 3531.

16 C. S. Trevisan, A. E. Orel and T. N. Rescigno, J. Phys. B: At., Mol. Opt. Phys., 2006, 39, L255.

17 C. Winstead and V. McKoy, J. Chem. Phys., 2006, 125, 074302.

18 S. Tonzani and C. H. Greene, J. Chem. Phys., 2006, 125, 094504.

19 D. Bouchiha, J. D. Gorfinkiel, L. G. Caron and L. Sanche, J. Phys. B: At., Mol. Opt. Phys., 2006, 39, 975.

20 M. Stepanoviæ, Y. Pariat and M. Allan, J. Chem. Phys., 1999, 110, 11376.

21 R. Dressler and M. Allan, Chem. Phys., 1985, 92, 449.

22 B. C. Ibănescu, O. May, A. Monney and M. Allan, Phys. Chem. Chem. Phys., 2007, 9, 3163.

23 A. Stamatovic and G. J. Schulz, Rev. Sci. Instrum., 1968, 39, 1752.

24 M. J. Frisch, G. W. Trucks, H. B. Schlegel, G. E. Scuseria, M. A. Robb, J. R. Cheeseman, J. A. Montgomery, Jr., T. Vreven, K. N. Kudin, J. C. Burant, J. M. Millam, S. S. Iyengar, J. Tomasi, V. Barone, B. Mennucci, M. Cossi, G. Scalmani, N. Rega, G. A. Petersson, H. Nakatsuji, M. Hada, M. Ehara, K. Toyota, R. Fukuda, J. Hasegawa, M. Ishida, T. Nakajima, Y. Honda, O. Kitao, H. Nakai, M. Klene, X. Li, J. E. Knox, H. P. Hratchian, J. B. Cross, V. Bakken, C. Adamo, J. Jaramillo, R. Gomperts, R. E. Stratmann, O. Yazyev, A. J. Austin, R. Cammi, C. Pomelli, J. Ochterski, P. Y. Ayala, K. Morokuma, G. A. Voth, P. Salvador, J. J. Dannenberg, V. G. Zakrzewski, S. Dapprich, A. D. Daniels, M. C. Strain, O. Farkas, D. K. Malick, A. D. Rabuck, K. Raghavachari, J. B. Foresman, J. V. Ortiz, Q. Cui, A. G. Baboul, S. Clifford, J. Cioslowski, B. B. Stefanov, G. Liu, A. Liashenko, P. Piskorz, I. Komaromi, R. L. Martin, D. J. Fox, T. Keith, M. A. Al-Laham, C. Y. Peng, A. Nanayakkara, M. Challacombe, P. M. W. Gill, B. G. Johnson, W. Chen, M. W. Wong, C. Gonzalez and J. A. Pople, GAUSSIAN 03 (Revision C.01), Gaussian, Inc., Wallingford, CT, 2004.

25 M. G. Curtis and I. C. Walker, J. Chem. Soc., Faraday Trans., 1992, 88, 2805.

26 A. Kühn, H. P. Fenzlaff and E. Illenberger, J. Chem. Phys., 1988, 88, 7453 .

27 T. Skalický and M. Allan, J. Phys. B, 2004, 37, 4849.

28 A. M. Scheer, P. Możejko, G. A. Gallup and P. D. Burrow, J. Chem. Phys., 2007, 126, 174301.

29 M. B. Robin, Higher Excited States of Polyatomic Molecules, Academic Press, New York, 1974, vol. 1.

30 M. B. Robin, Higher Excited States of Polyatomic Molecules, Academic Press, Orlando, 1985, vol. 1.

31 K. Kimura, S. Katsumata, Y. Achiba, T. Yamazaki and S. Iwata, Handbook of HeI Photoelectron Spectra of Fundamental Organic Molecules, Japan Scientific Societies Press, Tokyo, 1981.

32 Y. Pariat and M. Allan, Int. J. Mass Spectrom. Ion Processes, 1991, $103,181$.

33 L. G. Christophorou, J. G. Carter and A. A. Christodoulides, Chem. Phys. Lett., 1969, 3, 237.

34 C. D. Cooper, W. T. Naff and R. N. Compton, J. Chem. Phys., 1975, 63, 2752.

35 M. Lezius, Int. J. Mass Spectrom., 2003, 223-224, 447.

36 R. Dressler and M. Allan, J. Electron Spectrosc. Relat. Phenom., 1986, 41, 275.

37 H. D. Flosadóttir, S. Denifl, F. Zappa, N. Wendt, A. Mauracher, A. Bacher, H. Jónsson, T. D. Märk, P. Scheier and O. Ingólfsson, Angew. Chem., Int. Ed., 2007, 46, 8057. 